\title{
ESCRITURA ACADÉMICA DE ESTUDIANTES INDÍGENAS EN LA UNIVERSIDAD
}

\section{ACADEMIC WRITING BY INDIGENOUS STUDENTS AT UNIVERSITY}

\author{
Margareth Marmolejo Caicedo ${ }^{1}$ \\ Róbinson Grajales Alzate ${ }^{2}$ \\ Universidad del Valle, Cali, Colombia
}

\section{RESUMEN}

La escritura académica de estudiantes indígenas en contextos de bilingüismo e interculturalidad ha sido estudiada desde diferentes enfoques como las corrientes decoloniales, los programas para evitar la deserción universitaria y la enseñanza de la literacidad académica. Este artículo tiene como objetivo describir el discurso académico de estudiantes indígenas universitarios a través de textos argumentativos, con el fin de conocer los aspectos que deben ser priorizados en la

$1 \quad$ Magíster en Estudios Interlingüísticos e Interculturales de la Universidad del Valle. Candidata a doctora en lingüística de la Universidad de Antioquia. Docente de la Universidad del Valle. Actualmente es investigadora del equipo PRESEEA-Cali y pertenece al Grupo de Estudios Lingüísticos e Interdisciplinarios de la Universidad del Valle y al Grupo de Estudios Sociolingüísticos de la Universidad de Antioquia.

margareth.marmolejo@correounivalle.edu.co ORCID: https://orcid.org/0000-0003-4146-6125

2 Doctor en Lingüística de la Universidad de Antioquia. Profesor de lingüística teórica y descriptiva de la Escuela de Ciencias del Lenguaje de la Universidad del Valle, Colombia. Actualmente es el coordinador del equipo PRESEEA-Cali y dirige el proyecto Estudio sociolingüístico de Cali. Además, es director del Grupo de Estudios Lingüísticos e Interdisciplinarios de la Universidad del Valle, en el que se trabajan las líneas de investigación en lingüística hispánica, sociolingüística y lexicografía. robinson.grajales@correounivalle.edu.co

ORCID: https://orcid.org/0000-0002-8699-1321 enseñanza de la literacidad académica y cómo se lleva a cabo su aprendizaje. A partir de un corpus de textos argumentativos de estudiantes indígenas de primer semestre, provenientes de distintas comunidades y de programas académicos diferentes, se analizó su producción escrita en relación con cuatro categorías: la densidad léxica, la fragmentariedad, la superestructura y la intertextualidad. Se encontró que ninguno de los textos argumentativos cumple de manera adecuada las convenciones del discurso académico. Entre los aspectos que deben priorizarse para alcanzar la literacidad académica están la ampliación del léxico en español y del vocabulario técnico, la identificación y uso de recursos semánticos y discursivos para cohesionar y dar coherencia a las ideas del texto, la construcción de textos con la superestructura requerida y la incorporación de la polifonía con diferentes propósitos y con las convenciones establecidas para ello. 


\section{PALABRAS CLAVE}

literacidad, escritura académica, textos argumentativos, enseñanza de la escritura, estudiantes indígenas.

\section{ABSTRACT}

Academic writing from indigenous students have been studied in bilingual contexts and interculturality from different perspectives such as decolonization, programs aiming to stop school dropouts and the teaching of academic literacy. This paper intends to describe academic discourse from indigenous students at university through their writing of argumentative texts, in order to know what aspects should be prioritized when teaching academic literacy and how it should be learned. A corpus of argumentative texts from first-semester indigenous students from various ethnicities was analyzed in regard to four categories: lexical density, text fragmentation, superstructure and intertextuality. We found that none of the texts produced fulfill completely the requisites to be considered part of academic discourse. What has to be prioritized with the intention of achieving academic literacy is: to widen student's technical lexicon in Spanish, to identify and use discursive and semantic resources with the purpose of creating cohesion and give coherence to texts, to build texts with the correct superstructure and to include polyphony for different goals and with the accurate conventions demanded.

\section{KEY WORDS}

literacy, academic writing, argumentative texts, teaching writing, indigenous students.

\section{INTRODUCCIÓN}

Dentro del amplio ámbito de la escritura académica, uno de los asuntos que se han explorado en los últimos años es la relación de los estudiantes que provienen de comunidades minoritarias con este tipo de escritura en la universidad (Soler, 2012). Este tema ha cobrado importancia no solo desde la perspectiva de los estudios decoloniales (Caicedo y Castillo, 2008) sino también desde el análisis de las causas de la deserción universitaria, dentro de las cuales tiene un papel preponderante la dificultad para adquirir las prácticas letradas propias del discurso académico (Guzmán et al., 2009).

En diferentes contextos de bilingüismo $\mathrm{e}$ interculturalidad en el mundo se han hecho estudios sobre los obstáculos de estudiantes de comunidades indígenas para integrarse al discurso académico (Rose, Grace y Cowey, 1999; Nakata, 1999; Vigil, 2004; Hill, 2010; García Negroni y Hall, 2010; Olate y Henríquez, 2010; Bastiani-Gómez y López-García, 2016). Entre las dificultades sobresalientes evidenciadas en estas investigaciones se encuentran: la poca consciencia, por parte de los profesores, en la planeación de los procesos de alfabetización para los estudiantes que tienen como lengua materna una lengua minoritaria; la escasa articulación de la escritura académica con los contextos externos al escolar; las actitudes lingüísticas frente a la primera y la segunda lengua de los estudiantes indígenas; las diferencias en el nivel de bilingüismo entre estudiantes de una misma comunidad; la deficiente formación básica en procesos de literacidad; entre otros aspectos.

En Colombia, se han hecho algunos estudios sobre estos aspectos relacionados con la escritura académica de estudiantes indígenas (Corrales, 2011; Chaparro, 2011; Reyes, 2014), específicamente en el ámbito universitario (Agray, 2014; Bustamante, 2005; Cortés, 2007; Rocha, 2016; Mora, 2016; Meneses, 2011; Meneses y Castillo, 2011; Soler, 2012). En estas investigaciones se han señalado aspectos importantes como la influencia del nivel de bilingüismo de los estudiantes en el aprendizaje de la escritura académica (Corrales, 2011); la falta de una apropiación adecuada de la escritura y la lectura en la lengua materna, 
y en el español como segunda lengua en los casos de bilingüismo; y una relación entre la poca suficiencia en español académico, sumada a las herramientas insuficientes en algunas instituciones, y el bajo rendimiento académico y la deserción (Bustamante, 2005).

En este sentido, es importante avanzar en investigaciones que describan la escritura académica de los estudiantes indígenas en las universidades, con el propósito de ver cuáles son las principales barreras y sensibilizar sobre sus necesidades, así como sobre la importancia de incluir estos hallazgos en los contenidos curriculares. La investigación que presentamos aquí parte del análisis de textos de estudiantes indígenas universitarios para describir las características de su escritura académica en español.

Para ello, partimos del concepto de literacidad académica entendida como un conjunto de prácticas lingüísticas utilizadas para construir conocimiento y negociar la pertenencia a una comunidad académica o profesional (Colombi, 2015). Este lenguaje académico, en el ámbito universitario, adquiere unos propósitos y unas características que deben ser acogidas por los miembros de esa comunidad.

Según Teberosky (2007), la finalidad del discurso académico es explicar y argumentar con el fin de convencer y persuadir a una comunidad de la validez de ciertos presupuestos. Este propósito les daría unas características generales a todos los textos académicos, sin negar que también existen particularidades dentro de las culturas académicas (Owtram, 2010). Para Teberosky (2007) el discurso académico promueve la construcción de un texto propio a partir de la intertextualidad. Esos textos producen diferentes posturas enunciativas en el marco de unas prácticas académicas que inscriben al escritor en un contexto retórico.

Además de su propósito y la intertextualidad, los textos académicos se caracterizan por ser emitidos por un miembro de la comunidad académica, dirigidos a otros miembros que juzgan y evalúan el texto y al emisor; exponen un tema determinado con un contenido proposicional o referencial en un contexto que impone los cánones de lo que es apropiado o no (Halliday, 2014); pertenecen al registro formal; y tienen una organización determinada que exige elementos como la presentación por apartados y la inclusión de figuras, tablas, notas al pie, etc. (Teberosky, 2007).

Dentro del género discursivo universitario (Bajtín, 1982; Cassany, 2007; Mundó y Castelló, 2013) se han establecido los aspectos más relevantes que deben ser identificados y evaluados en los textos académicos: la superestructura prototípica con los componentes que se incluyen en la introducción, el desarrollo y la conclusión de los textos argumentativos, con sus respectivas fórmulas para marcar comienzos y finales (Van Dijk, 1980); las estrategias de condensación de la información que dan cuenta de la competencia conceptual y léxica del autor, dentro de las cuales están el uso de tecnicismos, la nominalización y otras formas de empaquetamiento (Halliday, 2014) que se pueden observar a través de la densidad léxica; el uso de conectores lógicos, referencias anafóricas y catafóricas, relaciones lógicas, entre otros elementos relacionados con la coherencia y la cohesión (Hoey, 2001); y la intertextualidad, que incluye los procedimientos y los propósitos de las citas (Teberosky, 2007), así como las convenciones gráficas para introducirlas.

De acuerdo con lo anterior, la densidad léxica, la fragmentariedad -que comprende los aspectos que rompen la coherencia y la cohesión- (Desinano, 2009), la superestructura y la intertextualidad serán definidas en la metodología como variables de análisis junto con los componentes que se observaron en cada una ellas. De esta manera, estas categorías 
funcionan como una referencia para establecer el nivel de adecuación de los textos de los estudiantes indígenas a las convenciones del discurso académico. Esta observación se hace a través del análisis de los errores identificados en el corpus textual estudiado.

El análisis de errores es una metodología que fue creada en el marco de la enseñanza de lenguas extranjeras, derivada del concepto de interlengua (Selinker, 1972), que se entiende como un sistema idiolectal del estudiante a través del cual se puede identificar el nivel de adquisición de una segunda lengua en el que se encuentra (Selinker, 1972). El punto de referencia para determinar el nivel es la lengua meta. La forma de establecerlo es por medio del análisis de errores, que permite observar las características de la producción oral o escrita de los estudiantes (Corder, 1981). Así se sabe la etapa en la que se encuentran los aprendices y a partir de estos datos se generan estrategias de aprendizaje. Los errores son sistemáticos, surgen del desconocimiento de la regla o la convención; por el contrario, las equivocaciones no son sistemáticas y se producen por falta de atención porque el estudiante conoce la norma (James, 1998).

En el caso de la escritura académica de estudiantes indígenas se toman como referencia de llegada las convenciones del discurso académico. Se analizan los errores de los estudiantes para describir su producción escrita y se establece cuáles son los elementos que faltan para adecuarse al discurso académico según la clasificación del tipo de errores. Con base en los datos obtenidos, se pueden planear las intervenciones de tipo pedagógico y didáctico para el aprendizaje de la escritura académica en la universidad por parte de los estudiantes indígenas.

A partir de estas consideraciones, se plantearon las preguntas que motivaron nuestra investigación: ¿Cuáles aspectos deben ser priorizados en la enseñanza de la escritura académica a los estudiantes indígenas en la universidad? ¿Qué indican los errores de los estudiantes indígenas en sus textos académicos sobre cómo llevan a cabo el aprendizaje de la literacidad académica?

De acuerdo con lo expuesto hasta aquí, el objetivo de esta investigación es describir el discurso académico de estudiantes indígenas universitarios a través del análisis de errores en textos argumentativos escritos.

Analizar los errores de los estudiantes indígenas que están en el proceso de inserción en la literacidad académica proporciona insumos para delimitar y comprender este proceso, así como para mejorar las prácticas de enseñanza y aprendizaje alrededor de la escritura en la universidad, con el fin de que sean relevantes y acordes con las necesidades de los alumnos indígenas. De igual manera, este trabajo puede sensibilizar sobre la necesidad de diseñar estrategias pedagógicas y didácticas adecuadas a cada grupo poblacional que habita la universidad, a partir de datos reales sobre su desempeño. En cualquier caso, un trabajo como el que presentamos incorpora un punto de vista que debe considerarse en el diseño de materiales y estrategias de enseñanza, así como en la planificación de las políticas institucionales para la inclusión de diferentes comunidades y tipos de estudiantes. Así mismo, sería una contribución en el camino hacia la comprensión de una de las causas de la deserción académica de los estudiantes indígenas, e incluso no indígenas: la dificultad para adquirir la literacidad propia del ámbito universitario.

\section{METODOLOGÍA}

Esta es una investigación descriptiva a partir de datos recogidos durante el primer semestre de 2017. El objetivo es describir y clasificar los errores más relevantes de la producción escrita de textos argumentativos de estudiantes 
indígenas universitarios. En el corpus textual analizado se localizaron, describieron y clasificaron los errores hallados para hacer una estadística descriptiva que permitiera observar su distribución en cada uno de los participantes.

Los participantes de esta investigación son cuatro estudiantes indígenas matriculados en el primer semestre en la Universidad del Valle en Cali, Colombia; pertenecen a diferentes programas académicos de pregrado, son de distintas etnias y tienen edades comprendidas entre los 16 y los 20 años. Inicialmente, se había convocado a 27 estudiantes indígenas del primer semestre matriculados en los grupos de español, pero la mayoría no participó aduciendo falta de tiempo o exceso de trabajo académico. Para caracterizar a los estudiantes que aceptaron participar se aplicó un cuestionario en el que se recogió información sociológica como etnia, edad, sexo, lenguas habladas, lenguas en las que han recibido educación formal, lugar de origen y lenguas habladas con los padres, lenguas habladas dentro de la universidad, entre otros aspectos que fueron tenidos en cuenta en análisis de los resultados. De esta manera, se establecieron sus principales características:

Tabla1. Participantes de la investigación.

\begin{tabular}{|c|c|c|c|c|c|c|c|}
\hline \multirow{2}{*}{ Participante } & \multirow{2}{*}{ Programa académico } & \multirow{2}{*}{ Sexo } & \multirow{2}{*}{ Pueblo } & \multicolumn{2}{|c|}{ Bilingüe } & \multicolumn{2}{|c|}{ Lugar de origen } \\
\cline { 5 - 8 } & & & Sí & No & Urbano & Rural \\
\hline 2HPM & Ingeniería topográfica & \multirow{2}{*}{ Hombre } & Pastos & & $X$ & & $X$ \\
\hline 1FNM & Ingeniería electrónica & Hombre & Pastos & & $X$ & & $X$ \\
\hline 2FMB & Trabajo Social & Mujer & Nasa & & $X$ & $X$ & \\
\hline
\end{tabular}

En el código del participante las letras corresponden a si es hombre $(H)$ o mujer $(F)$, a la inicial del pueblo al que pertenece (Pastos, Nasa o Misak) y a si son monolingües (M) o bilingües $(B)$.

El corpus de análisis está constituido por 16 textos argumentativos, 4 por cada participante, de entre 500 y 600 palabras cada uno. Como punto de partida para el ejercicio de escritura, a los participantes se les proporcionaron para la lectura dos textos de referencia, uno a favor y otro en contra, sobre las temáticas del calentamiento global, los estudios superiores y el lugar de los indígenas en la sociedad, así como un texto sobre la importancia de la ciencia en la humanidad. Se buscaba que con estos textos se desencadenara una polémica que les permitiera a los participantes argumentar, citar y desarrollar una postura enunciativa. A partir de estos textos se les dieron cuatro consignas en las que se les pidió argumentar su posición sobre: 1) la realidad del calentamiento global; 2) la necesidad de los estudios universitarios para tener un buen nivel de vida; 3) una campaña para la inclusión de más estudiantes indígenas en la universidad; y 4) la necesidad de la ciencia para la humanidad.

Dentro de la consigna, se les indicó a los estudiantes que sus escritos serían leídos en el Cabildo Universitario, donde tomarían en cuenta su posición para la estructuración de una postura consensuada dentro del mismo cabildo. 
Esto se hizo con el fin de que los estudiantes supieran que sus textos serían leídos por un público amplio y no sólo por los investigadores, lo que a su vez les da un carácter funcional a los textos de los participantes. Además, se les pidió que escribieran por lo menos 500 palabras por texto, para tener un mínimo de 2000 palabras por participante, una cantidad que, según Nelson (2010), es adecuada para reflejar significativamente la lengua de alguien.

Ya que se pretendía observar la competencia en escritura académica de los estudiantes como se daría en la universidad, se citó a los estudiantes en dos ocasiones distintas a una sala de cómputo y se les pidió que escribieran un texto argumentativo, tal como sucede en una situación de examen. El procesador de palabras a través del cual debían escribir tenía deshabilitada la opción de autocorrección, por lo que los estudiantes debían acudir solo a sus conocimientos ortográficos. Para los otros dos textos que debían escribir, se les dio un plazo de ocho días para su elaboración, teniendo en cuenta que gran parte de las producciones escritas en la universidad se dejan como tareas. Esto se hizo además con el fin de contrastar los resultados de los textos producidos en situación controlada con aquellos que se dejan como tarea. Estos últimos textos, al tener mayor tiempo para su escritura, revisión y corrección, podrían mostrar rasgos diferentes.

Las categorías de análisis que se usaron para identificar y clasificar los errores en el corpus textual son cuatro:

- La densidad léxica: indica la proporción de unidades léxicas en comparación con las unidades funcionales del texto (preposiciones, artículos, conjunciones, etc.) que no dan información de contenido. Aunque no es posible establecer cuál debe ser el porcentaje mínimo de densidad léxica aceptable de un texto académico (expositivo, informativo, argumentativo, etc.), se espera que supere el cincuenta por ciento (Johansson, 2008). Para establecer este valor, se toma el total de types, se divide entre el total de tokens y se multiplica por cien. Dentro de la densidad léxica también se tuvieron en cuenta el uso de tecnicismos y los errores de elección léxica y de procesos (verbos). Es importante aclarar que la densidad léxica no mide la legibilidad o complejidad de un texto, sino la cantidad de información.

- La fragmentariedad: incluye los aspectos que rompen la coherencia y la cohesión del texto y, por lo tanto, dificultan la lectura y la comprensión (Desinano, 2009). Los indicadores de fragmentariedad analizados en los textos de los participantes fueron puntuación, ortografía sobre palabras léxicas y procesos, problemas de relaciones lógicas y lógico-semánticas (Halliday, 2014), errores gramaticales, errores de referencias anafóricas y catafóricas, problemas de colocaciones y rasgos orales.

- La superestructura: la estructura formal que representa la distribución de los contenidos según el orden de aparición (Van Dijk, 1980). La superestructura es la que permite la construcción de la tipología textual y permite también reconocer funciones académicas. Para esta investigación se obtuvieron textos argumentativos cuya superestructura mínima corresponde a la introducción, el desarrollo de argumentos y la conclusión. Se analizó el porcentaje del total de palabras que representó cada parte en relación con el texto completo y si su presentación textual estaba determinada por una unidad textual de párrafo o no.

- La intertextualidad: hace parte de la función interpersonal descrita por Halliday (2014) y las citas son una referencia a investigaciones anteriores publicadas en otros textos (Teberosky, 2007). Las 
citaciones se relacionan con la atribución de contenido proposicional a otras fuentes dentro de la función ideacional. Así, las citas sirven para persuadir social y epistemológicamente, proveer justificación para los argumentos, demostrar la novedad de la posición propia, demostrar que el texto está en relación con el conocimiento y el contexto disciplinario, por lo que puede aportar algo.

Para el análisis de estos aspectos se siguieron diferentes procedimientos. En primer lugar, se calculó la densidad textual con la fórmula especificada antes en este mismo apartado. Para realizar esta operación, se recurrió al software Antconc, que permite contabilizar la totalidad de los types y tokens. En este primer momento, a partir de la lectura de los textos, se identificaron los tecnicismos y los errores de elección léxica. En segundo lugar, gracias al mismo software se contabilizaron y clasificaron los conectores lógicos, se analizó el uso de mayúsculas y minúsculas, de referencias anafóricas y catafóricas, y se hizo una descripción de la utilización de los signos de puntuación y la ortografía viendo su contexto y función dentro del texto. En tercer lugar, se buscó la superestructura de cada texto mediante la identificación de una introducción, discusión y conclusión. Sólo se describió si estos aspectos aparecían o no y cuál era su posición dentro del texto. Por último, se identificaron en cada texto las citas de los artículos de referencia que se les había dado a los estudiantes, con el fin de describir su manera de citar y corroborar el uso de la intertextualidad como recurso argumentativo y académico. Todos los elementos encontrados fueron etiquetados y clasificados para realizar los procedimientos cuantitativos.

Además del porcentaje de densidad léxica, se aplicaron fórmulas cuantitativas para obtener el promedio de errores en cada una de las categorías obtenidas a partir de la clasificación, así como los porcentajes de distribución de los componentes de la superestructura y la frecuencia absoluta de las citas y los tipos de citas en los textos. De esta manera, se obtuvieron los resultados que se presentan en el siguiente apartado.

\section{RESULTADOS}

Para la descripción de la escritura académica de los estudiantes indígenas que participaron se observaron los componentes de las categorías de análisis en los textos producidos por cada una de las consignas definidas en la metodología. A continuación, se presentan los resultados obtenidos en cada una de ellas.

\section{DENSIDAD LÉXICA}

En la tabla 2 se pueden observar los porcentajes de densidad léxica obtenidos en cada uno de los cuatro textos que se les pidió escribir a los estudiantes a partir de las cuatro consignas.

Tabla 2. Densidad léxica discriminada por participante y consigna

\begin{tabular}{|c|c|c|c|c|}
\hline & 1HPM & 2HPM & 1FNM & 2FMB \\
\hline Consigna 1 & 42,7 & 47,3 & 52,2 & 39,4 \\
\hline Consigna 2 & 43,0 & 45,0 & 44,7 & 43,7 \\
\hline Consigna 3 & 48,2 & 40,5 & 48,2 & 38,3 \\
\hline Consigna 4 & 46,6 & 45,4 & 48,3 & 42,4 \\
\hline
\end{tabular}


Se puede observar que la estudiante bilingüe (2FMB) fue quien obtuvo el menor porcentaje. Esta estudiante tuvo toda su educación en una institución bilingüe de la zona rural de Silvia, Cauca. 1FNM, la participante con la mayor densidad léxica, por su parte, tuvo una educación monolingüe en una institución no indígena en la ciudad de Cali, institución en la que le dedicaban dos horas semanales a la asignatura de español.

Dado que la densidad léxica está relacionada con los tecnicismos, se estableció el promedio de uso de estos términos, como se muestra en la siguiente figura:

Figura 1. Vocabulario técnico promedio.

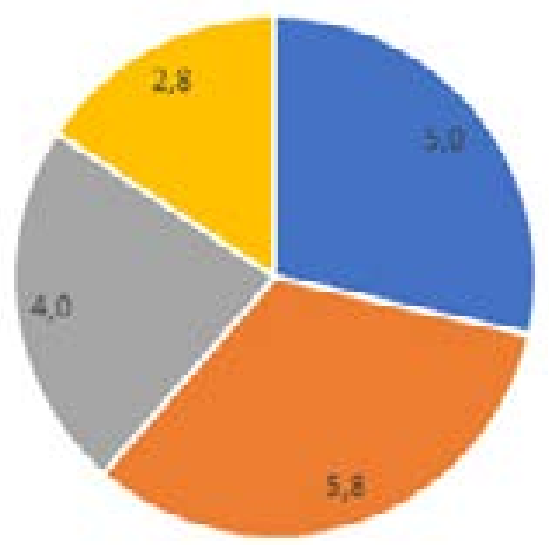

- $1 \mathrm{HPM}=2 \mathrm{HPM}=1 \mathrm{FNM}=2 \mathrm{FMB}$

La participante 1FNM, que tuvo mayor densidad léxica, fue la tercera participante que más tecnicismos utilizó, un promedio de cuatro palabras por texto. Los sujetos 1HPM y 2HPM, quienes tienen porcentajes de densidad léxica similares, utilizaron en promedio más palabras técnicas que el resto de las participantes. Así pues, el sujeto con mayor uso de tecnicismos, $2 \mathrm{HPM}$, utilizó la fórmula $\mathrm{CO}_{2}$ para referirse al dióxido de carbono, utilizó palabras y colocaciones como metano, efecto invernadero, gases contaminantes, oferta laboral, Pruebas Saber11, CERN, Bosón de Higgs y utilizó verbos como talary culminar sin repetición en los textos. Por lo contrario, la participante $2 \mathrm{FMB}$, quien tuvo en promedio 2.8 palabras técnicas por texto, utilizó palabras como, por ejemplo, sequías, oxígeno, ácido sulfúrico, fertilizante y el único verbo técnico utilizado fue emprender. Cabe la pena resaltar que esta participante repitió dentro de sus textos las palabras técnicas ya utilizadas y en la consigna 3 no utilizó ninguna.

Otras investigaciones (Bastiani-Gómez y López-García, 2016), en las que se obtuvieron resultados similares, han concluido que estos datos indican que el vocabulario empleado por los estudiantes es pobre y repetitivo.

No es posible determinar si la baja densidad léxica y el poco uso de tecnicismos de la participante $2 \mathrm{FMB}$ se deban a su condición bilingüe, entre otras cosas porque no hay más sujetos bilingües en la investigación. Sin embargo, en el cuestionario de caracterización, 2FMB afirmó que no se sentía muy cómoda leyendo periódicos porque había vocabulario desconocido que no le permitía comprender los 
artículos. Además, esta participante aseguró que durante su educación media dedicaban entre una y dos horas semanales al aprendizaje formal del español, mientras que los otros participantes aseguraron haber visto entre 2 y 4 horas semanales. El sujeto 2 HPM, siendo quien recibió más horas de español, fue además quien mayor uso de tecnicismos presentó.

En cuanto a los errores de elección léxica y elección de proceso (verbo), se encontraron los datos recogidos en la figura 2 :

Figura 2. Promedio de errores de elección lexical y de proceso.

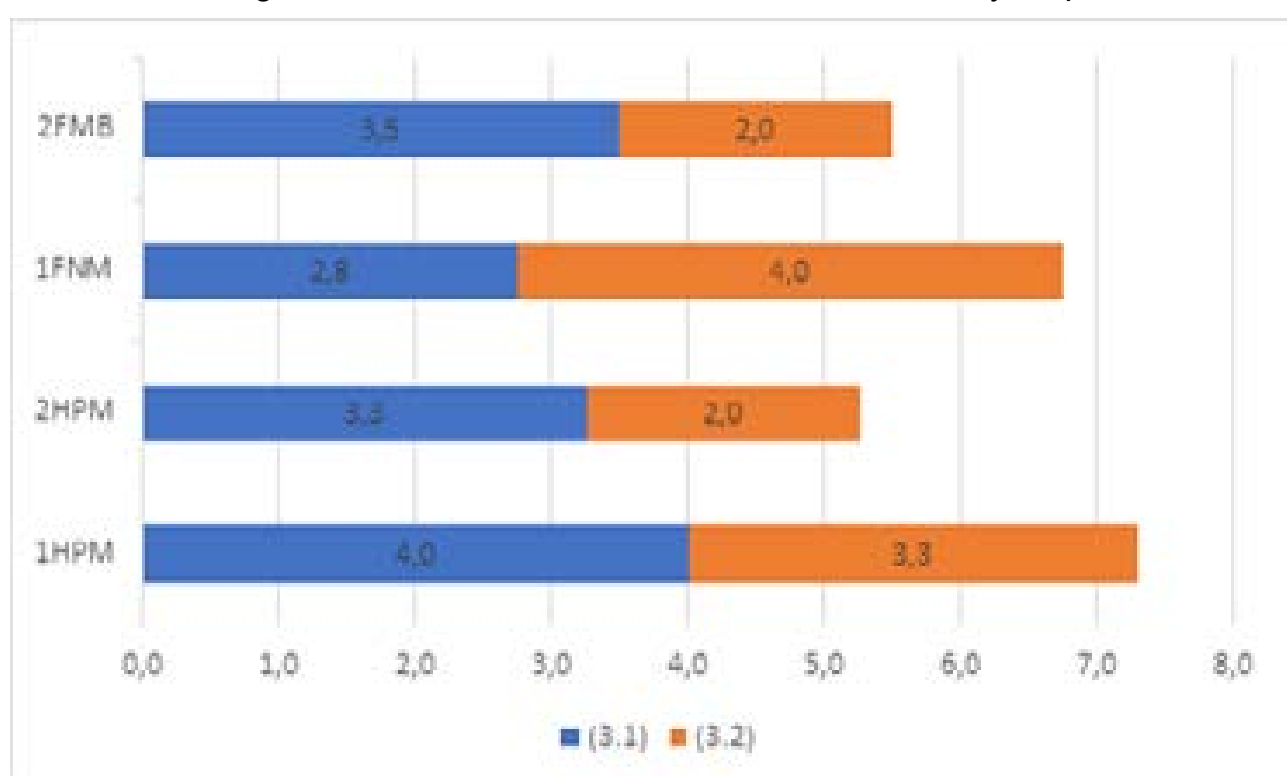

Se puede ver que los sujetos $1 \mathrm{HPM}$ y $2 \mathrm{HPM}$ obtuvieron el promedio más alto de errores en el momento de la elección léxica, en tanto que las participantes $1 \mathrm{FNM}$ y $2 \mathrm{FMB}$ tienen el promedio más bajo, aunque no por mucho. No obstante, los valores cambian en lo que respecta a los errores de elección verbal. 1HPM y 1FNM, ambos monolingües, emplearon mal un promedio de
2,3 y 3,8 procesos, respectivamente. Esto nos sugiere que la participante bilingüe $2 \mathrm{FMB}$ y el participante 2HPM tuvieron el promedio más bajo de los dos errores: 2.6 y 2.8 errores de elección léxica y de procesos por texto.

En la siguiente tabla se pueden leer algunos ejemplos de los errores cometidos por los participantes. 
Tabla 3. Ejemplos de errores de elección léxica y de proceso.

\begin{tabular}{|c|c|c|c|}
\hline 1HPM & 2HPM & 1FNM & 2FMB \\
\hline \multicolumn{4}{|c|}{ Errores de elección léxica } \\
\hline $\begin{array}{l}\text { - Consigna } 1 \\
\text { “el recurso hídrico } \\
\text { a nivel mundial } \\
\text { y hoy en día ha } \\
\text { descendido de una } \\
\text { manera directa” } \\
\text { • Consigna } 2 \\
\text {.... las diferentes } \\
\text { carreras de nuestro } \\
\text { país entra en una } \\
\text { marca de alta } \\
\text { competición en } \\
\text { cuanto al empleo...” } \\
\text { “...por la demanda } \\
\text { de un profesional la } \\
\text { empresa adquiere } \\
\text { más gastos...” }\end{array}$ & 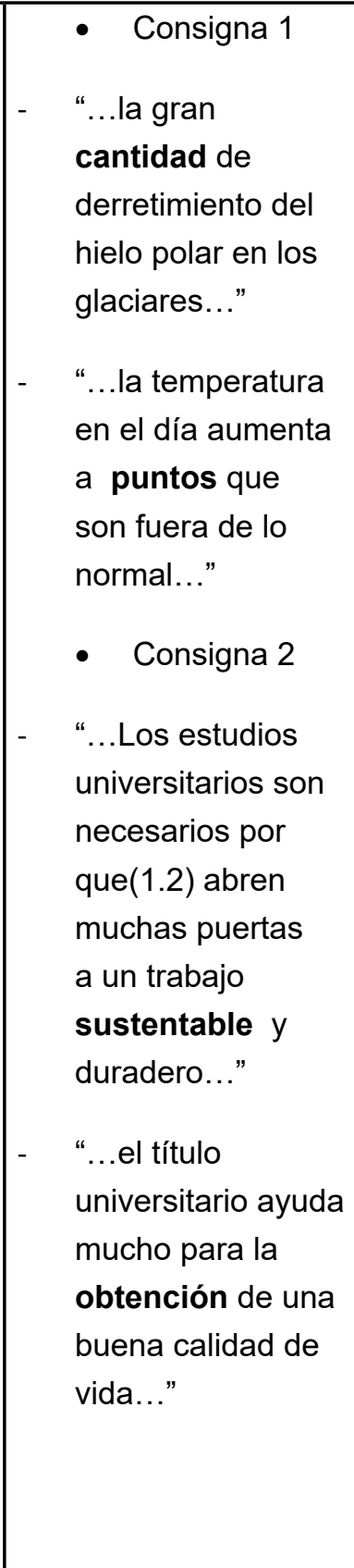 & $\begin{array}{l}\text { - Consigna } 1 \\
\text { - “..."History } \\
\text { ha perdido } \\
\text { credibilidad tanto } \\
\text { en el modo de su } \\
\text { programación...” } \\
\text { - Consigna } 2 \\
\text { - “...uno de los } \\
\text { mayores casos } \\
\text { por los que los } \\
\text { universitarios no } \\
\text { consiguen trabajo } \\
\text { es...” } \\
\text { - Consigna } 3 \\
\text { “...llegan } \\
\text { nuevos tipos de } \\
\text { tendencia y estas } \\
\text { ingresan en las } \\
\text { comunidades...” } \\
\text { - Consigna } 4 \\
\text { “...la ciencia ha } \\
\text { avanzado tanto } \\
\text { que nos facilita } \\
\text { la vida cotidiana } \\
\text { y ha llegado a } \\
\text { tal facilidad que } \\
\text { las personas se } \\
\text { conforman...” }\end{array}$ & \begin{tabular}{|l} 
- $\quad$ Consigna 1 \\
“...cuando vemos \\
ya el recorrido de \\
las aguas vemos \\
que ya no son \\
puras...” \\
• Consigna 2 \\
“...por falta de \\
necesidad, \\
buscas un trabajo \\
distinto, que no \\
se relaciona con \\
lo que habías \\
estudiado...” \\
• Consigna 3 \\
“...el ácido \\
sulfúrico sirve y \\
es buena para los \\
campos de los \\
fertilizante...” \\
• Consigna 4 \\
“...que así \\
podemos \\
mantener más \\
puramente la \\
cultura...”
\end{tabular} \\
\hline
\end{tabular}


Errores de elección de proceso

\begin{tabular}{|c|c|c|c|}
\hline $\begin{array}{l}\text { a. Consigna } 2 \\
\text { “...en nuestro diario } \\
\text { vivir y los retos que } \\
\text { se nos afrontan en } \\
\text { el transcurso de la } \\
\text { educación...” } \\
\text { b. Consigna } 4 \\
\text { “...un nuevo camino } \\
\text { lo lleva a uno a } \\
\text { entrar a nuevos } \\
\text { descubrimientos...” }\end{array}$ & $\begin{array}{l}\text { c. Consigna } 2 \\
\text { - “...algunas } \\
\text { industrias } \\
\text { clasifican otras } \\
\text { habilidades que } \\
\text { no las enseñan } \\
\text { en algunas } \\
\text { universidades...” } \\
\text { d. Consigna } 3 \\
\text { “...la falta de } \\
\text { conocimiento } \\
\text { que les impide } \\
\text { entender con } \\
\text { rapidez y claridad } \\
\text { los temas que } \\
\text { se ven en las } \\
\text { diferentes carreras } \\
\text { universitarias , por } \\
\text { eso surgieron } \\
\text { soluciones a estos } \\
\text { problemas...” }\end{array}$ & $\begin{array}{l}\text { e. Consigna 1 } \\
\text { - “...El } \\
\text { calentamiento } \\
\text { global es algo } \\
\text { tan complejo } \\
\text { que ha llevado } \\
\text { varios tipos de } \\
\text { investigación y no } \\
\text { podemos definir } \\
\text { todo...” } \\
\text { f. Consigna } 3 \\
\text { “...los estudiantes } \\
\text { así con estos } \\
\text { buscar y analizar } \\
\text { he dar cambios...” }\end{array}$ & $\begin{array}{l}\text { g. Consigna } 4 \\
\text { “...son las personas } \\
\text { quienes la[la ciencia] } \\
\text { dejan ver que es } \\
\text { mala...” } \\
\text { h. Consigna } 1 \\
\text { "...si el calentamiento } \\
\text { global se está dando } \\
\text { por todo el mundo y } \\
\text { es más intenso que } \\
\text { antes...” }\end{array}$ \\
\hline
\end{tabular}

Dentro de los hallazgos en la categoría de densidad léxica, se puede observar que el sujeto 2HPM, quien tuvo mayor número de horas de enseñanza de español como asignatura durante el bachillerato, mostró el abanico más amplio de palabras técnicas y tuvo el menor promedio de errores entre elección léxica y de proceso. De manera diferente, la participante 2FMB, bilingüe y que recibió entre una y dos horas por semana de la asignatura de español, obtuvo el menor porcentaje de densidad léxica y uso de tecnicismos, pero fue la segunda participante con menos errores tanto de elección léxica como elección de procesos. La participante $1 F N M$, siendo indígena por filiación pero que ha vivido toda su vida en una ciudad y nunca ha recibido educación en zona rural o indígena, obtuvo el mayor porcentaje de densidad léxica y el promedio más alto en el uso de tecnicismos. En cuanto a la elección léxica y de procesos, esta participante obtuvo un promedio de cuatro errores de elección de procesos, el promedio más alto, pero al mismo tiempo, obtuvo el promedio más bajo de error de elección léxica.

\section{FRAGMENTARIEDAD}

Los aspectos de fragmentariedad analizados en los textos de los participantes fueron puntuación, ortografía de las palabras, problemas de taxis, errores gramaticales, errores de referencias, problemas de colocaciones y rasgos orales. Estos aspectos serán presentados aquí en el mismo orden.

\section{PUNTUACIÓN}

En los textos de los participantes se marcaron los errores correspondientes a la puntuación no estándar y a la falta de puntuación obligatoria (no se marcó la puntuación facultativa según la 
Ortografía de la lengua española, publicada por la RAE en 2010). Este tipo de errores superaron considerablemente a otros dentro de la categoría de fragmentariedad. En promedio, todos los textos presentaron 26,2 errores de puntuación. Los más frecuentes fueron la no marcación del punto y de la coma, como se muestra en los siguientes ejemplos (entre paréntesis aparece el código con el que fueron marcados los errores en el lugar en el que se identificaron):

\section{No marcación de la coma:}

- "Buenos día(1.1) comunidad universitaria y comunidad encargada en de idiomas" [1HPM].

- “...el calentamiento global no existe(1.1) ya que según mis ideas y tomando como referencia el primer texto..." [1HPM].

- "...algo que aumenta lo real que es el calentamiento global es que en países donde hay más industrialización(1.1) sobre todos las potencias mundiales..." [2HPM].

- "Los dos textos tienen muy buenos fundamentos para dar a entender su posición(1.1) pero en mi punto de vista..." [1FNM].

- "En primer lugar(1.1) la frase "los estudios universitarios son necesarios para tener un buen nivel de vida" [2FMB].

- "El mundo exterior también nos ha ayudado a que tengamos una buena, no excelente(1.1) pero una buna vida, si la sabemos manejar y controlar(1.1) estoy segura de que un pueblo indígena nunca se acabara" [2FMB].

\section{No marcación del punto:}

- “...porgran ambición del enriquecimiento de los viene s nos lleva a destruir nuestro propio mundo(1.1) además de ello tanto los unos como los demás somos los responsables" [1 HPM].

- "Buenos día comunidad universitaria y comunidad encargada en las área de idiomas(1.1) Según los textos que me ofrecieron" [1HPM].

- "...el efecto invernadero que es el principal causante del calentamiento global(1.1)" [final de párrafo] [2HPM].

- “...el programa se basa más en entregar preguntas que en resolver estas)(1.1) por otro lado national geographic ha sido un canal que muestra varios" [1FNM].

- “...tubos huecos insertados en el barro para recoger los sedimentos de años atrás y hasta los anillos de los árboles,(1.1) la investigación que habla sobre que le calentamiento global no existe..." [1FNM].

En la puntuación no estándar también aparecieron como errores frecuentes el espacio tipográfico después del paréntesis y las comillas de apertura, así como espacio antes de punto seguido. Este error apareció sobre todo en el sujeto 2HPM, que de manera sistemática dejó un espacio tipográfico entre palabra y signo de puntuación. Veamos algunos ejemplos:

- "...son las ranas ( animales anfibios existentes en mi lugar de origen hace varios años)" [1HPM].

- “...basándose solo en caparazones de almejas gigantes y muestras de coral 
, mientras que national geographic..." [1FNM].

- “...se ve más claramente el efecto que produce el calentamiento global : por ejemplo estados unido..." [2HPM].
- “...pueden o han creado cosas sorprendentes, como los demás lo han hecho y como lo harán. Los pueblos indígenas también..." [2FMB].

En la siguiente figura vemos la cantidad de errores cometidos por los participantes en cada consigna.

Figura 3. Errores de puntuación.

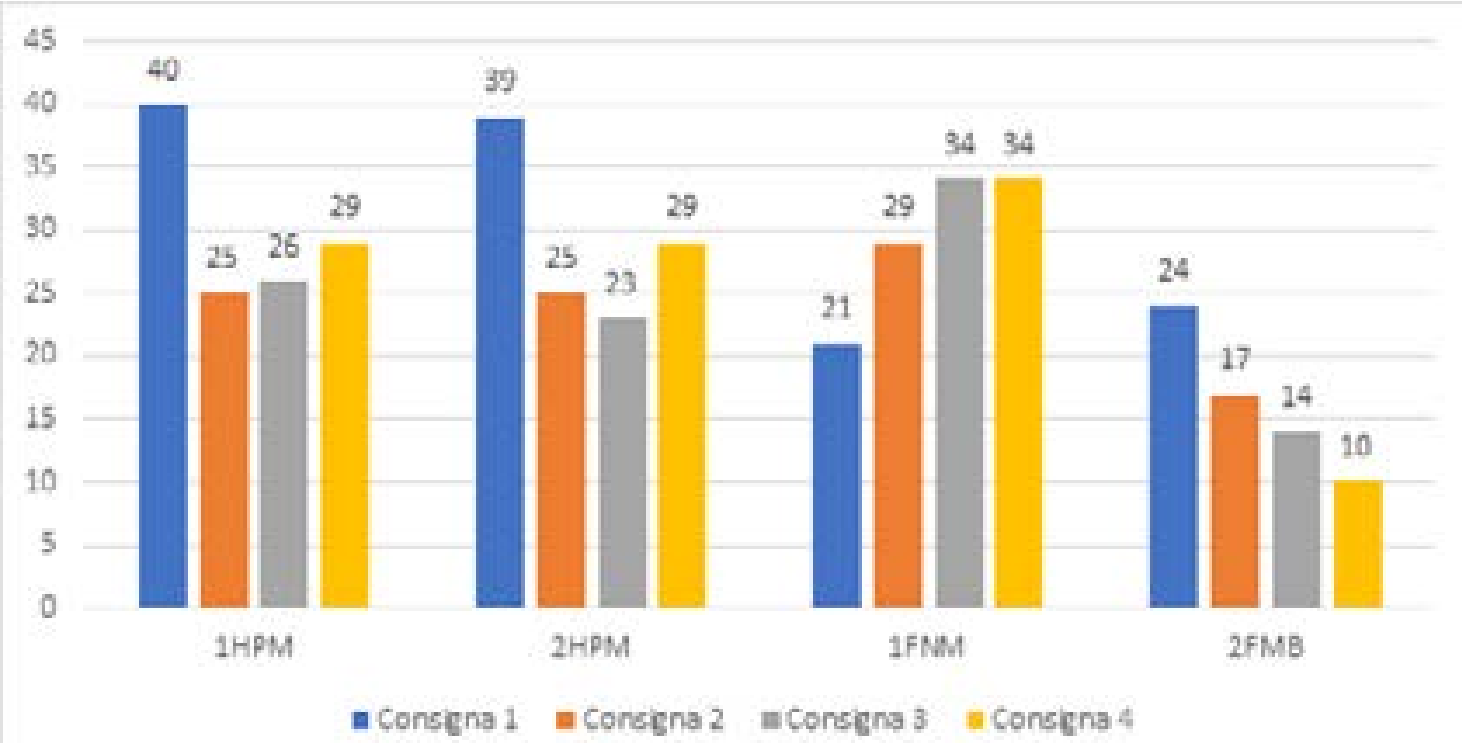

Gracias a la figura 3 podemos ver que no hay una tendencia única sobre la consigna con más errores. Pero es importante resaltar que la participante bilingüe $2 \mathrm{FMB}$ fue quien menos presentó errores de puntuación. En la tabla 4, vemos el promedio de errores de puntuación por participante.

\begin{tabular}{|c|c|c|c|c|}
\hline Tabla 4. Promedio de errores de puntuación \\
& 1HPM & 2HPM & 1FNM & 2FMB \\
\hline Promedio & 30 & 29 & 29,5 & 16,3 \\
\hline
\end{tabular}

No es posible encontrar una explicación fundamentada sobre por qué la participante 2FMB tiene el promedio más bajo de errores de puntuación. Una hipótesis que debe ser comprobada con más sujetos bilingües misak es que $2 F M B$, al tener dos códigos con reglas de puntuación casi idénticas, tal vez es más consciente de las reglas.

\section{Ortografía de las palabras}

Los errores de ortografía relacionados con la escritura de las palabras aparecieron en promedio 5,6 veces por texto. En la figura 4 se puede ver el promedio de errores de cada participante en las cuatro consignas. 
Figura 4. Errores de ortografía de las palabras.

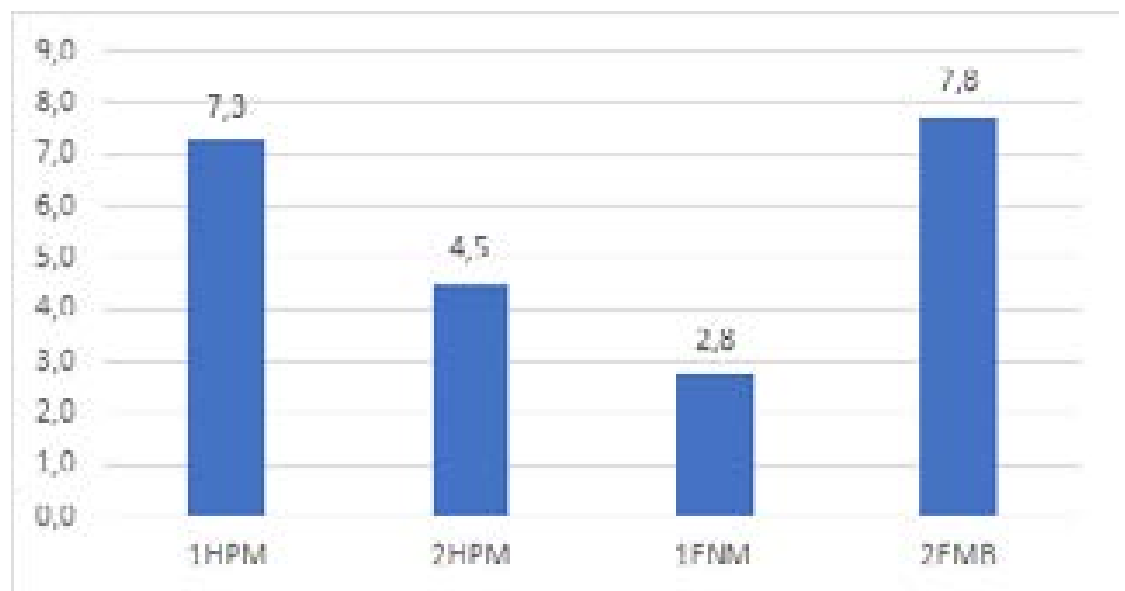

Contrariamente a lo sucedido con la puntuación, para la ortografía la participante $2 \mathrm{FMB}$ tuvo el promedio más alto de errores cometidos con 7,8 errores por texto. La sigue el sujeto 1HPM quien fue también el participante con mayor promedio de errores de puntuación. Luego el sujeto 2HPM con 4,5 errores en promedio y luego, con un promedio muy bajo en comparación con los otros participantes, está 1FNM con aproximadamente 2,8 errores por texto. Este resultado no parece explicarse por algún factor social o de bilingüismo. A continuación, se presentan algunos ejemplos de este tipo de errores:

- “...porgran ambición del enriquecimiento de los viene $\mathbf{s}$ [bienes] nos lleva a destruir nuestro propio mundo..." [1 HPM].

- "Los estudios universitarios son necesarios por que [porque] abren muchas puertas a un trabajo sustentable" [2HPM].

- “...en algunos lugares como lo es áfrica hay escases [escasez] de agua por este gran fenómeno" [1FNM].

- "La ciencia en sí, para mi [mí] es buena, sino que son las personas quienes la dejan..." [2FMB].

El tipo de error ortográfico más común fue la incorrecta separación o unión de palabras, incluso más que la ausencia o equivocada colocación de tildes. La participante bilingüe fue quien más errores tuvo a la hora de separar las palabras, produjo algunos sistemáticos como por que (cuando no debía separarse), des de, a hora, a una así, mientras que los participantes monolingües solo fueron sistemáticos con por que.

Aparte de la incorrecta separación de palabras, otros errores encontrados en todos los participantes fueron la omisión y el uso incorrecto de la $h$; la omisión de la tilde en los adverbios y pronombres interrogativos, en las formas verbales del pasado, en la partícula sí en locuciones adverbiales y en el adverbio de afirmación; tildes en palabras que no la tienen; posibles errores de digitación como los cambios de $b$ por $v, z$ por $s$, o por $u$; elisión de letras en diferentes contextos como la $d$ final en calidad o facilidad; y flexiones incorrectas en nombres y verbos.

Otro error sobresaliente fue el uso no estándar de mayúsculas y minúsculas. En el caso de las mayúsculas, se encuentran luego de coma para iniciar un nuevo enunciado, en palabras que no son nombres propios y para hacer énfasis, como el caso de "NO" utilizado por uno de los participantes. En cuanto a las minúsculas, aparecen al inicio de párrafos, después de puntos seguidos y en nombres propios. 
La participante bilingüe es quien más errores de ortografía presenta, sobre todo en la separación de palabras. Sin embargo, es quien menos errores de puntuación presentó y la única que no cometió ningún error de uso de mayúsculas y minúsculas. Los hablantes monolingües presentan errores de puntuación y de ortografía de las palabras en general, lo que no parece estar relacionado con la etnia o la cantidad de horas estudiadas de español en la educación básica.

\section{ERRORES DE DEPENDENCIA LÓGICA Y RELACIONES LÓGICO-SEMÁNTICAS}

El sistema de taxis, desde una perspectiva funcional (Halliday, 2014), corresponde a las relaciones de interdependencia lógica y las relaciones lógico-semánticas entre complejos de cláusulas. Este sistema es de gran importancia para la realización de la metafunción textual, pues permite establecer la jerarquía entre las ideas. Cuando este sistema falla, es difícil comprender el alcance o la relación lógica de una idea escrita en relación con otra. Las relaciones de interdependencia lógica pueden ser de dos tipos, de parataxis (coordinación) o de hipotaxis (subordinación). Las relaciones lógico-semánticas muestran el tipo de relación conjuntiva entre dos o más cláusulas.

Los errores de taxis son tal vez los errores más complejos en los textos estudiados. Este tipo de error afecta directamente la coherencia del texto. En el siguiente ejemplo vemos cómo el participante 1HPM propone cuatro cláusulas sin relación lógica entre ellas dentro de un complejo de cláusulas:

III en cuanto a mis posturas ideológicas sobre el calentamiento global [[donde se me presento

1 2

la siguiente cuestión]] || de acuerdo a los dos textos ofrecidos con temas relacionados con el

3

calentamiento global y sus evidencias \|y otro donde evidencia que el calentamiento global

4

no existe. |||

La primera es una cláusula no finita que no está en relación de dependencia con alguna de las otras tres. La segunda cláusula, por el contrario, es sintácticamente dependiente de la primera por una relación de hipotaxis, aunque en realidad, semánticamente es una cláusula independiente que intenta introducir "una cuestión", pero vemos que inmediatamente sigue la tercera cláusula que hace referencia a dos textos sobre las evidencias del calentamiento global y no a una cuestión en particular. La cuarta cláusula parece referirse a un tercer texto que muestra que el calentamiento global no existe, pero el estudiante tuvo acceso solo a dos textos, uno con evidencias a favor de la existencia del calentamiento global y otro con evidencia sobre la no existencia del fenómeno, es decir que no hubo un tercer texto.

En la siguiente figura podremos ver el promedio de errores de taxis cometidos por cada participante. 
Figura 5. Errores de taxis.

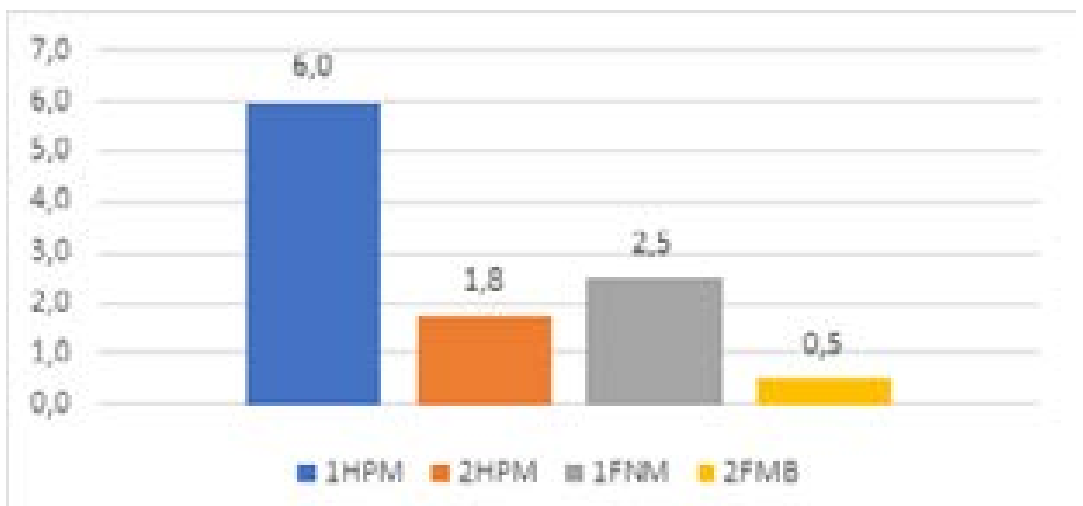

Es interesante ver cómo los estudiantes 1HPM y $1 F N M$ son aquellos con el mayor promedio de errores de taxis. 2HPM y $2 \mathrm{FMB}$, por su parte, tienen el menor promedio. Especialmente la participante $2 \mathrm{FMB}$ fue quien menos errores cometió. Esta participante tuvo un solo error por cada dos textos. Tanto el estudiante que tuvo más errores de taxis (1HPM) como la que menos presentó (2FMB) fueron quienes recibieron menos horas de español por semana en su formación básica. Lo único que diferencia a la participante $2 \mathrm{FMB}$ del resto de participantes es que ella es bilingüe. Por el contrario, 1HPM solo se diferencia por haber sido el sujeto monolingüe que menos horas de enseñanza de español recibió durante la educación media.
Entre los errores de las relaciones lógico semánticas y de dependencia lógica, se encuentran los siguientes (entre paréntesis aparece el código con el que fueron etiquetados en los textos): los errores de finito con consecuencia lógica (2.2), error de frase preposicional (2.3), error de cohesión por elipsis o sustitución (2.5), error de concordancia entre sujeto y finito (2.6), error de concordancia entre determinante y nombre (2.7), error de cohesión por conjunción (2.8), error de referencia anafórica (2.9), error de concordancia entre el nombre y el adjetivo (2.10), y error de determinante (2.11). Todos estos elementos rompen la coherencia y la cohesión del texto. En la siguiente figura se muestra el promedio de estos errores por cada participante.

Figura 6. Otros errores que rompen la coherencia y la cohesión.

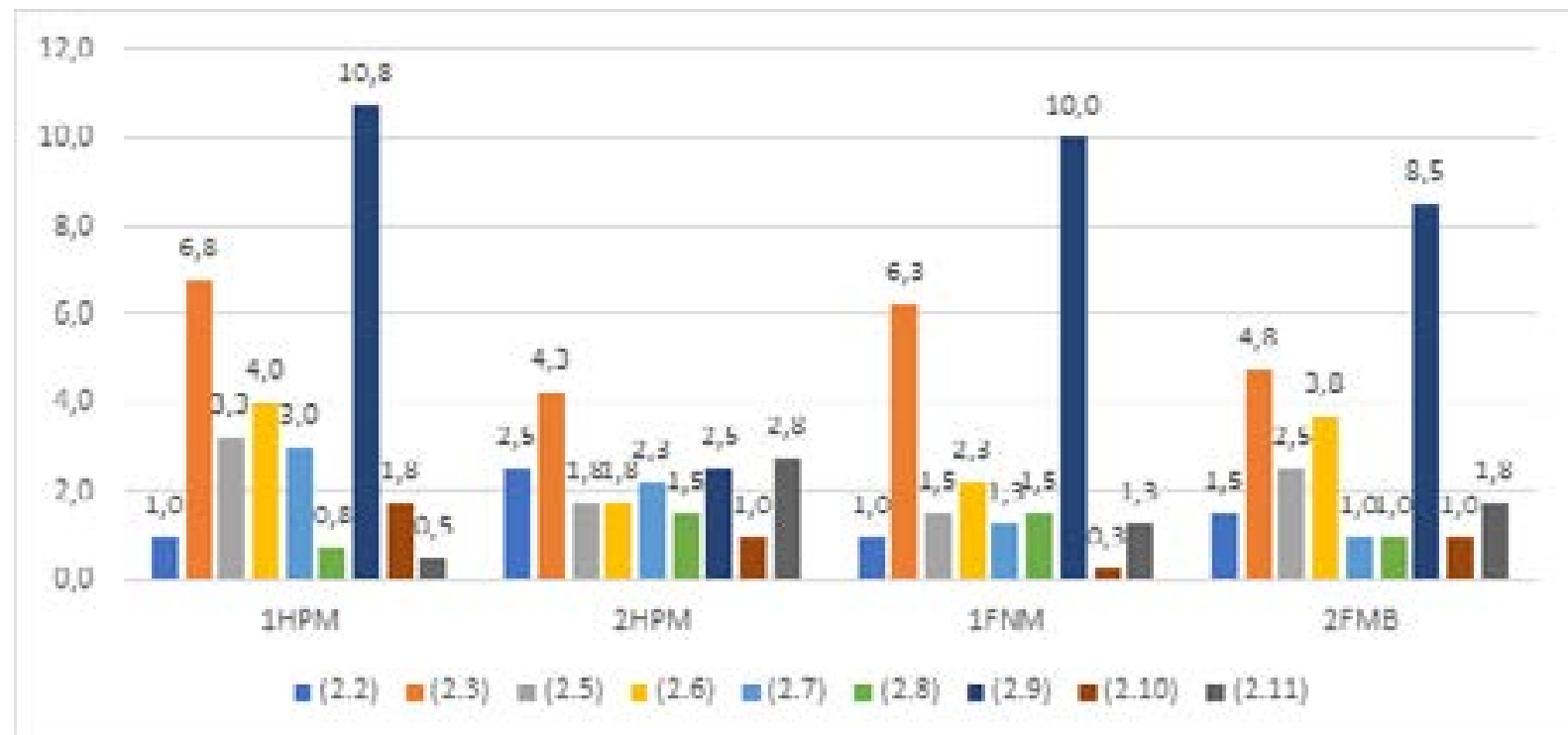


Aquí se observa que el error de coherencia y cohesión más común en los textos es el de referencia anafórica (2.9). Casi siempre se presentó cuando se introducía un sujeto en forma de pronombre que no había sido mencionado antes. El siguiente error más frecuente es el de frase preposicional. Este se dio porque los participantes utilizaban la preposición equivocada para el verbo principal o por una colocación no estándar. El caso más común fue de acuerdo a en vez de de acuerdo con. Asimismo, en este error fue común la falta de la preposición que exigía el verbo.

También se puede ver que el sujeto 2HPM fue quien tuvo el menor promedio de errores en general. Le sigue la participante $1 \mathrm{FNM}$, luego 2FMB y, por último, con un porcentaje de errores más alto, el sujeto 1HPM. Podemos pensar que el bajo número de errores se debe a que 2HPM es el participante que dijo haber recibido la mayor cantidad de horas de instrucción sobre la lengua materna. No obstante, no muy lejos de él está $1 \mathrm{FNM}$ y la participante bilingüe, quienes tuvieron menos horas de instrucción. Aunque $1 \mathrm{HPM}$ haya tenido la misma cantidad de horas de instrucción que $2 \mathrm{FMB}$ y $1 \mathrm{FNM}$, tuvo una educación rural y no es bilingüe.

\section{OTROS ASPECTOS}

Otros aspectos no estándares que aparecieron en los textos y que hacen que los mismos no se inscriban en el contexto universitario son los rasgos orales (4.1), la redundancia (4.2), el error de expresión idiomática (4.4) y el error de registro (4.5).

La figura 7 nos permite ver los promedios de aparición de estos aspectos en cada participante.

Figura 7. Otros aspectos no estándares.

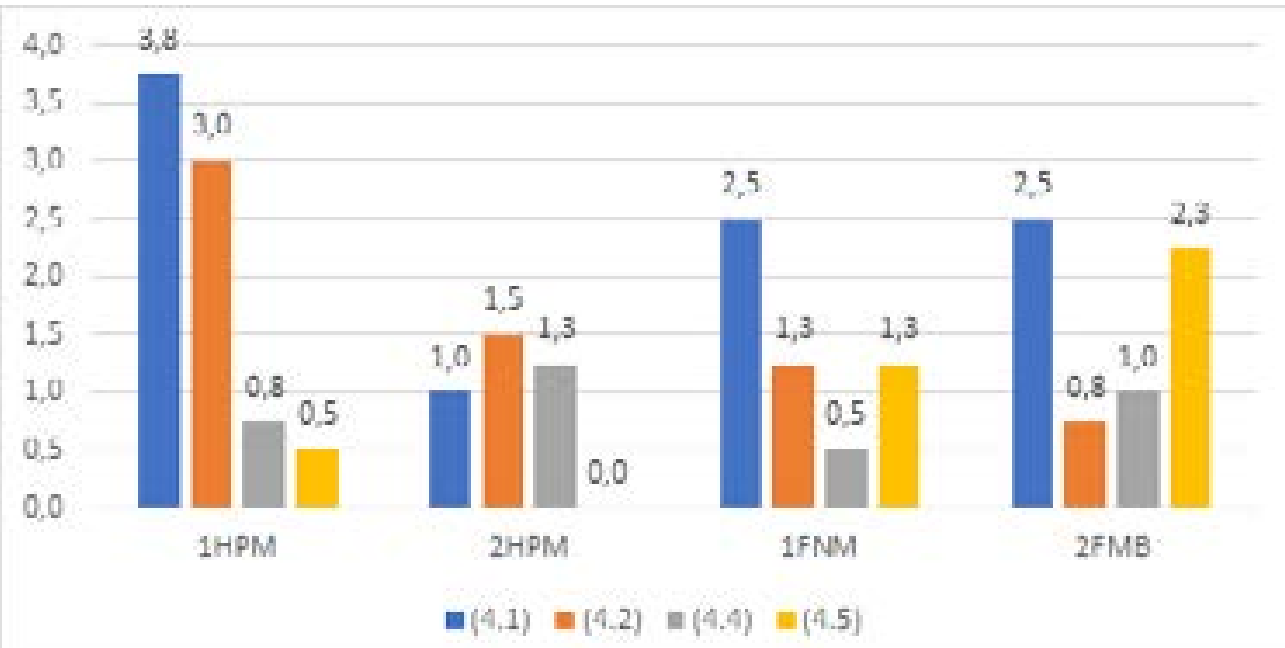

El rasgo oral más común en todos los participantes fue el dequeísmo. También el uso de léxico común en la oralidad, pero no en la escritura, además del uso de lo que es, lo duro es.

La redundancia estuvo marcada por el uso innecesario de doble pronombre como en "...en nuestro entorno en donde vivimos ha sufrido cambios" o “...en mi periodo de vida de mi infancia viví mi infancia con ciertos animalitos".

El error de colocación se dio sobre todo en la expresión "tanto de" y "entre". Por ejemplo: "...que existimos y que podamos llegar a tener un nivel de conocimiento como ellos, y así ayudarnos entre sí [mutuamente]". Otro ejemplo: “...nos lleva a destruir nuestro propio mundo además de ello tanto los unos como 
los demás [tanto los unos como lo otros] somos los responsables de esta crisis".

El error de registro se debió a la utilización del pronombre de la segunda persona del singular informal o familiar tú para referirse al lector, aspecto que se evita en la academia, en la que impera la segunda persona del singular formal usted.

\section{OBSERVACIONES GENERALES SOBRE LA FRAGMENTARIEDAD}

Se podría afirmar que la educación explícita sobre la lengua materna definitivamente juega un rol importante en el resultado de la escritura, si comparamos los resultados entre sujetos monolingües. No obstante, podríamos argumentar también que, en el caso de la participante bilingüe, la cantidad de horas de estudio de español recibidas durante la educación media solo tuvo un impacto negativo en lo relacionado con la densidad léxica, el uso de tecnicismos y la correcta elección léxica. Para los aspectos mencionados, el desempeño de la participante bilingüe estuvo por debajo del desempeño de los otros participantes. En cambio, su desempeño en lo relacionado con la fragmentariedad, es decir la coherencia y la cohesión, estuvo marcado por promedios de errores bajos, incluso menores que los de los sujetos monolingües.

De igual manera, se podría aseverar que no hay tampoco mucha diferencia entre los sujetos 2HPM y 1FNM, aunque ambos recibieron una cantidad diferente de horas de enseñanza del español y provienen de contextos diferentes. El primero viene de una zona rural y la segunda de una zona urbana. Lo único en lo que la participante 1FNM superó positivamente a 2 HPM fue en la densidad léxica. No siendo así en lo que respecta a la fragmentariedad, el sujeto 2HPM fue quien menos errores cometió en general, exceptuando el error de puntuación. La participante 1FNM tuvo promedios de errores ligeramente más altos que 2HPM. Podríamos pensar, entonces, que provenir de una zona rural, pero con un alto número de horas de enseñanza del español puede superar por poco a la educación monolingüe con menos horas de enseñanza del español en una zona urbana, al menos en lo que respecta a la fragmentariedad.

Finalmente, podríamos decir de manera no concluyente, también, que el bilingüismo no parece ser el rasgo predominante a la hora de escribir textos con fragmentariedad. Y que este fenómeno se presenta independientemente de la lengua materna, la cantidad de horas de español estudiadas en el bachillerato y de la zona de proveniencia de los participantes. Sin embargo, podemos aclarar que en lo que respecta a los sujetos monolingües, la cantidad de horas estudiadas sí influye sobre el promedio de errores cometidos.

Además, es importante resaltar que el hecho de haber producido dos consignas en situación controlada y otras dos en situación no controlada no representó ningún resultado significativo, ya que en algunos casos la mayor cantidad de errores fueron producidos en la situación no controlada, como se puede ver en la tabla 5 .

\begin{tabular}{|l|c|c|c|c|}
\hline \multicolumn{5}{|c|}{$\begin{array}{c}\text { Tabla 5. Comparación de promedio de errores } \\
\text { en situación controlada y no controlada }\end{array}$} \\
\hline & 1HPM & 2HPM & 1FNM & 2FMB \\
\hline $\begin{array}{l}\text { Situación } \\
\text { controlada }\end{array}$ & 4,4 & 3,3 & 3,2 & 2,7 \\
\hline $\begin{array}{l}\text { Situación } \\
\text { no } \\
\text { controlada }\end{array}$ & 4,2 & 3,1 & 3,9 & 3,1 \\
\hline
\end{tabular}

\section{SUPERESTRUCTURA}

Los estudiantes produjeron textos argumentativos cuya superestructura mínima corresponde a la introducción, el desarrollo de argumentos y la conclusión. Se analizó, a partir de la cantidad de palabras, el porcentaje que representó cada parte en relación con la 
totalidad del texto y si su presentación estaba determinada por una unidad textual de párrafo o no. A continuación, presentamos el análisis de la superestructura en los cuatro textos producidos por cada participante.

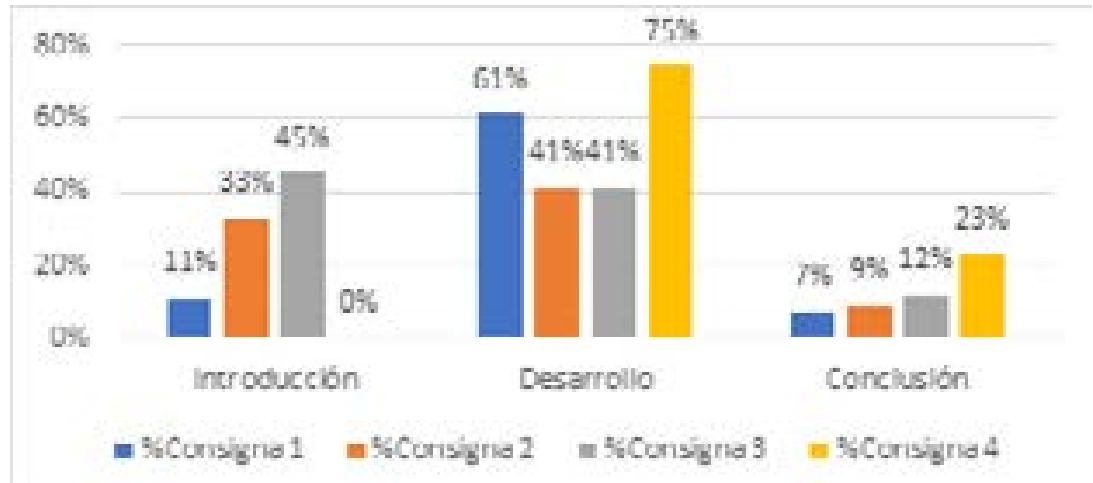

Figura 8. Análisis de la superestructura 1HPM.

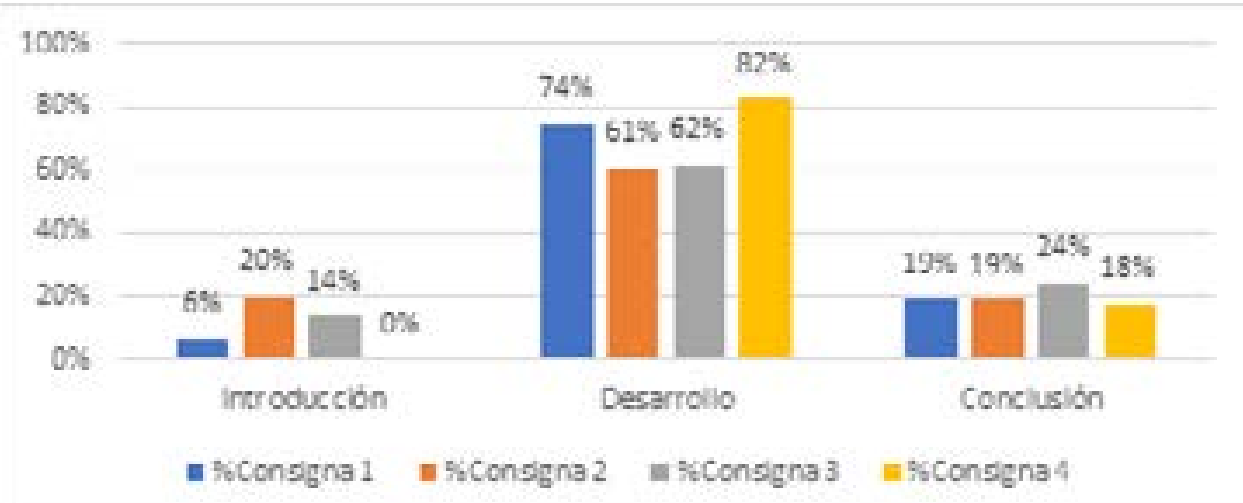

Figura 9. Análisis de la superestructura 2HPM.

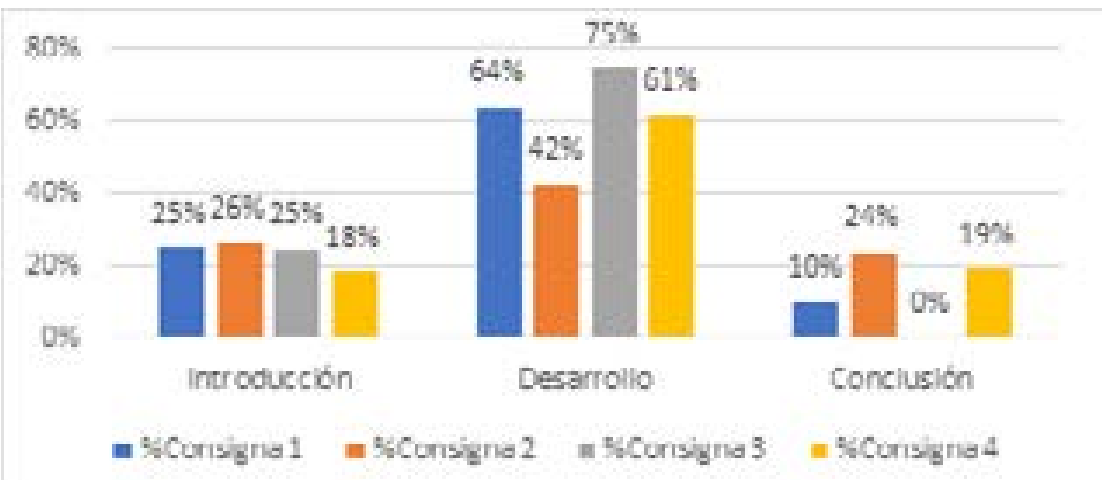

Figura 10. Análisis de la superestructura 1FNM. 


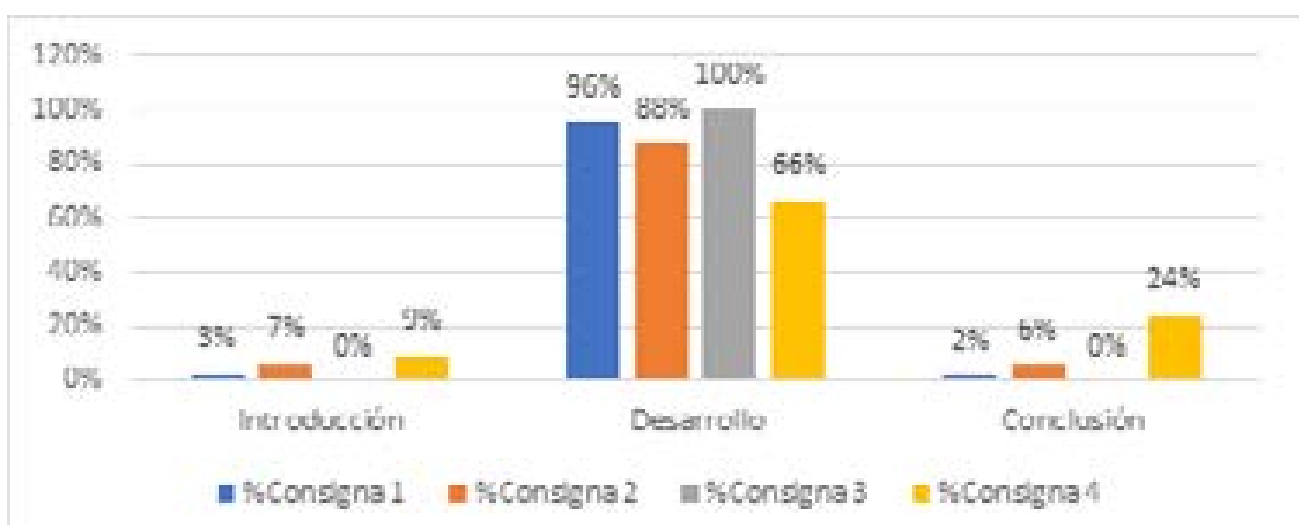

Figura 11. Análisis de la superestructura 2FMB.

El sujeto 1HPM escribió en promedio cuatro párrafos por texto. En general, tuvo en cuenta las tres partes esperadas de la superestructura, con excepción de la consigna 4 en la que no se observó introducción. Vemos en la figura 8 que la conclusión ocupó el menor porcentaje del texto, mientras que el desarrollo de los argumentos ocupó en promedio la mitad del texto.

Es necesario recordar que las consignas 1 y 4 se produjeron en situación controlada. Se podría pensar que al tener menos tiempo para escribir y estar en una situación de presión, estas consignas podrían mostrar menor desarrollo de argumentos. Pero sucede lo contrario. En dichas consignas, el sujeto 1HPM mostró una extensión mayor para el desarrollo de argumentos que en las consignas 2 y 3 , realizadas en casa.

Con respecto a la presentación textual de cada apartado, 1HPM presentó en general la introducción dentro del primer y segundo párrafo junto con el desarrollo de argumentos. Solo en la consigna 3 el primer y segundo párrafo correspondían solamente a la introducción. Sobre la conclusión se puede decir que este participante mostró tendencia a presentarla en un párrafo independiente y a introducirla con una frase de conclusión. No obstante, en la consigna 3 la conclusión se presentó a mitad del segundo párrafo y en la última oración del tercer párrafo.

Se puede decir, entonces, que para el participante
1HPM la superestructura no estuvo dividida en párrafos de manera sistemática y que no hay una tendencia sobre el porcentaje que debe representar cada parte en la superestructura total.

La superestructura en los textos del participante 2HPM tuvo un comportamiento similar a la del sujeto $1 \mathrm{HPM}$ en lo que respecta al desarrollo de los argumentos, como se aprecia en la figura 9. Vemos que en 2HPM las consignas 1 y 4 tienen un porcentaje de desarrollo mayor que en las consignas 2 y 3 . Aunque el valor porcentual no es igual en los participantes 2HPM y 1HPM, ambos obtuvieron casi el mismo porcentaje de desarrollo de argumentos en las consignas 2 y 3.

También se observa que 2HPM tuvo un porcentaje relativamente constante en lo que concierne a la conclusión y que solo varió en la consigna 3. La introducción, por el contrario, no tuvo un comportamiento estable $\mathrm{y}$, al igual que para el sujeto $1 \mathrm{HPM}$, estuvo ausente en la consigna 4.

Ahora bien, a diferencia del participante 1HPM, 2HPM fue sistemático con la ubicación de la introducción, el desarrollo y la conclusión. Este último participante siempre dividió la superestructura en párrafos. El primer párrafo, con excepción de la consigna 4, presentó siempre la introducción. Los párrafos posteriores 
estuvieron destinados al desarrollo de argumentos y el último párrafo a la conclusión. El promedio de párrafos escritos por este participante fue de 6 .

En la figura 10 se advierte que la superestructura de los textos de la participante 1FNM presentó un comportamiento diferente del de los dos participantes anteriores. En primer lugar, 1FNM fue sistemática con la proporción de la introducción en sus textos, mientras que los sujetos 1HPM y $2 \mathrm{HPM}$ no lo fueron. Nuevamente, el comportamiento de esta participante difiere totalmente del de los otros dos en que ella no tuvo valores porcentuales similares en el desarrollo de argumentos en las consignas 2 y 3 . 1FNM tuvo porcentajes similares en el desarrollo de argumentos de las consignas $1 \mathrm{y}$ 4, que correspondieron a las producciones en condición controlada.

En lo que respecta a la división de la superestructura en párrafos, 1FNM no mostró una tendencia evidente en la división de párrafos. Esto ya que la consigna 2 solo tiene un párrafo en el que aparecen todos los elementos de la superestructura. En la consigna 1, la introducción está presentada en los dos primeros párrafos del texto en lugar de un solo párrafo.

Aunque esta participante no haya sido sistemática con la división por párrafos, es posible decir que sí fue sistemática en el orden de aparición de las partes de la superestructura. La introducción, el desarrollo de argumentos y la conclusión fueron presentados siempre en este orden. Ya vimos que no ocurrió así en los textos del sujeto $1 \mathrm{HPM}$.

La participante 2FMB tuvo resultados bastante diferentes en comparación con los de los demás participantes. Es posible observar en la figura 11 que el porcentaje más alto en los textos estuvo ocupado por el desarrollo de argumentos. Las consignas 1 y 3 estuvieron constituidas casi exclusivamente por el desarrollo. La introducción fue el componente de la superestructura que menor porcentaje tuvo en las cuatro consignas. No muy lejos de este resultado estuvo de igual manera la conclusión.

Esta participante fue la que menos párrafos tuvo por consigna; en promedio, escribió dos. Al igual que la participante 1FNM, 2FMB presentó la información siempre iniciando con la introducción, seguida del desarrollo de argumentos y por último la conclusión.

Ninguno de los participantes fue sistemático con la organización textual, pues no mantuvieron la misma superestructura en las cuatro consignas, a pesar de que todos los textos eran argumentativos. No obstante, 2HPM, 1FNM y $2 F M B$ sí fueron sistemáticos en lo que respecta a la presentación de la introducción, el desarrollo de argumentos y la conclusión, al menos en el orden, aunque hay que reconocer que los tres participantes obviaron alguna de las partes en una de las consignas. En resumen, la superestructura de los textos argumentativos es una característica que no aparece claramente en los textos argumentativos de los estudiantes indígenas.

\section{INTERTEXTUALIDAD}

Las citaciones se relacionan con la atribución de contenido proposicional a otras fuentes dentro de la función ideacional. Así, las citas sirven para persuadir social y epistemológicamente, proveer justificación para los argumentos, demostrar la novedad de la posición propia, demostrar que el texto está en relación con el conocimiento y el contexto disciplinario, por lo que puede aportar algo. En la tabla 6 se puede apreciar el número de citas hechas por cada participante en cada consigna. 


\begin{tabular}{|c|c|c|c|c|}
\multicolumn{5}{|c|}{ Tabla 6. Número de citas por consigna } \\
\hline & 1HPM & 2HPM & 1FNM & 2FMB \\
\hline Consigna 1 & 3 & 0 & 5 & 1 \\
\hline Consigna 2 & 3 & 0 & 2 & 0 \\
\hline Consigna 3 & 1 & 0 & 2 & 0 \\
\hline Consigna 4 & 1 & 0 & 3 & 0 \\
\hline Promedio & 2,0 & 0,0 & 3,0 & 0,3 \\
\hline
\end{tabular}

Los participantes 1HPM y 1FNM son los únicos participantes que hicieron citas de manera sistemática en sus textos. En general, el tipo de cita que se privilegia, según el propósito, es aquella que sirve para proveer justificación a los argumentos, como en los siguientes ejemplos:

- "tomando como referencia el primer texto(4.6) donde nos evidencia que en nuestro entorno en donde vivimos ha sufrido cambios tales como el cambio de temperatura, el bajo nivel de los ríos" [1HPM].

- "no fueron suficientes los argumentos que dio history (científicos de la academia de china)(4.6) como para tomar esta en cuenta" [1FNM].

- "El primer texto (Juan Pablo Ruiz) (4.6) habla de que debemos proteger esos pueblos aislados que están adentrados" [1FMN].

La prevalencia de este tipo de citas muestra que se desconocen, o se conocen de manera precaria, otros propósitos con los que pueden ser empleadas, tales como iniciar una interrogación heurística, dar fidelidad a lo dicho por el autor o calificar, entre otros (Teberosky, 2007).

El sujeto $1 \mathrm{HPM}$ no mencionó las fuentes que citó y se refirió a ellas como "textos" que a su vez corresponden a los documentos a favor y en contra con los que contaban para escribir. Esta forma de citación no es estándar y deja desconcertado al lector que no ha leído los mismos textos. La forma en la que este sujeto cita hace pensar que él asume que sus lectores comparten su lectura, aun cuando las consignas indicaban que los textos producidos serían utilizados en contextos amplios con lectores diferentes. Además, cita ideas de los artículos sin darle crédito a los autores. Por el contrario, la participante 1FNM sí mencionó la fuente en once de doce citas. Esta participante menciona sea directamente al autor del artículo o a la fuente de publicación, atribuyéndole a la fuente el carácter de autor.

La participante 2FMB utilizó la expresión dicen para introducir otras voces en sus textos, fórmula en la que no se reconoce si hace referencia o no a los autores de los artículos de referencia. Esta estudiante, junto con 1HPM, son quienes citan de manera menos estándar, mientras que la participante 1FNM hace citaciones más directas y menciona a los autores, aunque sus citas no son estándares para el sistema universitario y sus producciones escritas no cuentan con apartado de referencias. En el caso del participante 2HPM no se encontró ninguna cita de los textos de referencia.

En resumen, los estudiantes no reconocen los propósitos de las citas ni las formas estándares de introducirlas en los textos argumentativos, según las convenciones del género discursivo académico. García Negroni y Hall (2010) denominan esta ausencia de citas para introducir proposiciones ajenas como distorsiones 
enunciativas, pues no comunican de manera clara al lector quién dijo qué, cuándo y dónde.

\section{CONCLUSIONES}

El primer aspecto que se constata en el análisis es que ninguno de los textos argumentativos de los participantes se inscribe de manera plena en el género discursivo de la universidad. En el cuestionario de caracterización, todos los estudiantes declararon que el aprendizaje de la literacidad académica era el asunto que les había causado mayor dificultad en su vida universitaria. Esto indica que se deben iniciar, de manera temprana, procesos de alfabetización académica explícita, teniendo en cuenta los elementos hallados en este trabajo como asuntos a abordar y que pueden coincidir o no con las necesidades de otros grupos de estudiantes que no sean indígenas.

En este sentido, según los resultados, en la enseñanza de la escritura académica a los

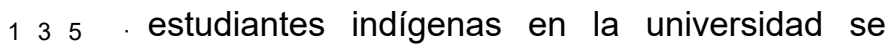
deben priorizar aspectos como la ampliación y la incorporación de léxico relacionado con los temas sobre los que se va a escribir, la identificación del vocabulario técnico y su uso en los textos académicos, y la elección del léxico adecuado para expresar las ideas de manera clara. También se debe privilegiar la enseñanza de las reglas de puntuación, la consciencia sobre la forma de las palabras y su relación con el sentido en la ortografía, los recursos semánticos y discursivos para cohesionar y dar coherencia a las ideas del texto, especialmente las concordancias y las correferencias, así como el reconocimiento de los elementos del discurso formal académico y la limitación de rasgos orales en su producción. De igual manera, es necesario el aprendizaje de la identificación de la superestructura de los textos de referencia y su visualización en la producción de los propios. Así mismo, se deben conducir aprendizajes sobre la identificación de la polifonía en los textos, los propósitos con los que se introduce y las convenciones establecidas para ello, con el fin de que los estudiantes incorporen estos elementos en su escritura académica.

Todos estos aspectos fueron identificados tanto en los textos que fueron producidos en situación controlada como no controlada, lo cual muestra que la situación de escritura no influyó en el desempeño y que, en algunos casos, los errores se produjeron más en la situación no controlada, donde se esperaban menos por la mayor disponibilidad de tiempo y la posibilidad de usar herramientas en la construcción de los escritos.

Si bien a través del análisis de errores se pudieron observar rasgos comunes en los textos de todos los participantes, particularmente la falta de adecuación al tipo de texto argumentativo académico y los errores de cohesión y coherencia, también se encontraron diferencias entre los participantes, como, por ejemplo, el dispar conocimiento sobre las reglas ortográficas, el dominio léxico y el uso de la intertextualidad. Estas diferencias podrían indicar que las características individuales de los estudiantes indígenas influyen en su aprendizaje de la literacidad académica. De esta manera, sería importante, en la alfabetización académica en el ámbito universitario, identificar y tener en cuenta en la enseñanza la comunidad de origen de los estudiantes indígenas, su nivel de bilingüismo y de español como segunda lengua o lengua materna y las condiciones de formación básica en español como las horas semanales dedicadas a esta materia en las instituciones educativas.

Los hallazgos de esta investigación ayudan a crear consciencia sobre las necesidades diferenciales en los procesos de alfabetización académica de estudiantes indígenas, las cuales deberían ser tenidas en cuenta en la planificación lingüística de las universidades, en la planeación de los microcurrículos, así como en la creación de instrumentos adecuados para afianzar el 
progreso académico de estos estudiantes, y de esta manera promover su permanencia en el sistema universitario, lo que aportaría, al lado de otros recursos, a mitigar la deserción universitaria de este grupo poblacional.

De todas maneras, habrá que hacer otros estudios para afianzar estas consideraciones, sobre todo ampliando el número de participantes, con el fin de que los resultados sean más abarcadores y generalizables a la población estudiantil indígena en el ámbito universitario.

\section{REFERENCIAS BIBLIOGRÁFICAS}

Agray, N. (2014). Minorías étnicas, interculturalidad y escritura académica en la Pontificia Universidad Javeriana. En C. García, S. Moya, H. Ramírez, J. Reyes, W. Sánchez y N. Agray. Reflexiones sobre lengua, etnia $y$ educación. Bogotá: Siglo del Hombre, pp. 227-262.

Bajtín, M. (1982). Estética de la creación verbal. México: Siglo XXI.

Bastiani-Gómez, J. y López-García, M. (2016). La producción de textos escritos en estudiantes de dos universidades públicas con el modelo intercultural. Revista Educación 40(1), 89-112.

Caicedo, J. y Castillo, E. (2008). Indígenas y afrodescendientes en la universidad colombiana: nuevos sujetos, viejas estructuras. Cuadernos Interculturales, 6 (10), 62-90.

Díaz, Á. (2014). Retórica de la escritura académica. Pensamiento crítico y argumentación discursiva. Medellín: Universidad de Antioquia.

Cassany, D. (2007). Análisis de una práctica letrada electrónica. Páginas de Guarda, 2, 99-112.
Colombi, M.C. (2015). Academic and Cultural Literact for Heritage Speakers of Spanish: a Case Study of Latin@ Students in California. Linguistic and education, 32, 5-15.

Corder, S. (1981). Error Analysis and Interlanguage. Oxford: Oxford University Press.

Cortés, P. (2007). Indígenas universitarios en la Universidad. Revista Etnias y Políticas. Observatorio Indígena de Políticas Públicas de Desarrollo y Derechos Étnicos, 4, 116-121.

Desinano, N. (2009). Los alumnos universitarios y la escritura académica. Análisis de un problema. Rosario: Homo Sapiens.

García Negroni, M. y Hall, B. (2010). Escritura universitaria, fragmentariedad y distorsiones enunciativas propuestas de prácticas de lectura y escritura focalizadas en la materialidad lingüísticodiscursiva. Boletín de Lingüística, 22(34), 41-69.

Guzmán, C., Durán, D., Franco, J., Castaño, E., Gallón, S., Gómez, K. y Vásquez, J. (2009). Deserción estudiantil en la educación superior colombiana. Metodología de seguimiento, diagnóstico y elementos para su prevención. Bogotá: Ministerio de Educación Nacional.

Halliday, M.A.K. (2014). An Introduction to Functional Grammar (4a ed.). Londres: Edward Arnold.

Hoey, M. (2001). Textual Interaction: An Introduction to Written Discourse Analysis. Londres: Routledge.

James, C. (1998). Errors in Language Learning and Use. Exploring Error Analysis. Londres: Routledge. 
Johansson, V. (2008), Lexical diversity and lexical density in speech and writing: a developmental perspective. Working Papers, 53, 61-79.

Meneses, A. (2011). Factores asociados a la deserción de estudiantes que ingresaron por condición de excepción indígena a la Universidad del Valle en 2011 - II. Sociedad y Economía, 40, 69-98.

Meneses, A. y Castillo, L. (2011). Estudio sobre los factores asociados a la deserción entre estudiantes afrocolombianos e indígenas de la Universidad del Valle. Cali: Universidad del Valle.

Mora, G. E. (2016). Dos experiencias educativas y editoriales con estudiantes indígenas, afrodescendientes $y$ de municipios pobres en la universidad desde una perspectiva intercultural. Forma $y$ Función, 29(1), 61-80.

Mundó, A. C., y Castelló, M. (2013). La escritura académica en la universidad. Revista de Docencia Universitaria, 11, 1-20.

Nakata, M. (1999). History, Cultural Diversity \& English Language Teaching. En Wignell, P. (Ed.). Double Power: English Literacy and Indigenous. Camberra: Education. Australian National Languages and Literacy.

Nelson, M. (2010) Building a written corpus. What are the basics? En O'Keeffe, A. y McCarthy, M. (eds.). The Routledge Handbook of Corpus Linguistics. Londres: Routledge.

Olate, A. \& Henríquez, M. (2010). Actitudes lingüísticas de profesores mapuche de Educación Básica: vigencia y enseñanza del mapudungun en el contexto educativo. Literatura y lingüística, 22, 103-116.
Owtram, N. T. (2010). The pragmatics of academic writing: A relevance approach to the analysis of research article introductions. Bern: Peter Lang.

Reyes, J. (2014). Una Revisión del Concepto de Escritura Académica en Situaciones de Bilingüismo e Interculturalidad. En C. García, S. Moya, H. Ramírez, J. Reyes, W. Sánchez y N. Agray. Reflexiones sobre lengua, etnia y educación. Bogotá: Siglo del Hombre, pp. 109-142.

Rose, D., Gray, B. y Cowey, W. (1999). Scaffolding Reading and Writing for Indigenous Children in School. En Wignell, P. (Ed.). Double Power: English Literacy and Indigenous. Camberra: Education. Australian National Languages and Literacy.

Sánchez, W. (2014). Políticas lingüísticas y programas educativos de las minorías étnicas en la universidad colombiana. En C. García, S. Moya, H. Ramírez, J. Reyes, W. Sánchez y N. Agray. Reflexiones sobre lengua, etnia $y$ educación. Bogotá: Siglo del Hombre, pp. $19-54$.

Selinker, L. (1972). Interlanguage. International Review of Applied Linguistics in Language Teaching, 10(1-4), 209-232.

Soler, S. (2013). Representaciones de la escritura académica en contextos de bilingüismo e interculturalidad. Signo $y$ Pensamiento, 32(62), 64-80.

Teberosky, A. (2007). El texto académico. En M. Castelló (Ed.). Escribir y comunicarse en contextos científicos y académicos: Conocimientos y estrategias. Madrid: Grao, pp. 17-46. 
Van Dijk, T. (1980). Texto y contexto. Madrid: Catedra.

Vigil, N. (2004). Acciones para desarrollar la escritura en lenguas indígenas. Glosas didácticas, 12, 174-183. 